\title{
Measurements of the Ion Species of Cathodic Arc Plasma in an Axial Magnetic Field
}

\author{
Efim Oks ${ }^{1}$ and André Anders ${ }^{2 *}$ Fellow \\ ${ }^{1}$ High Current Electronics Institute, 2/3 Academichesky Ave, Tomsk, 634055, Russia \\ ${ }^{2}$ Lawrence Berkeley National Laboratory, 1 Cyclotron Road, Berkeley, California, 94720, \\ USA
}

October 19, 2010

\section{ACKNOWLEDGMENT}

We are grateful to Dr. Georgy Yushkov, HCEI Tomsk, for his suggestions and comments. We thank Joe Wallig for technical assistance. The work was supported by the IPP project LBNL-T2-0196, funded by the Department of Energy under contract AC02-05CH11231.

\section{DISCLAIMER}

This document was prepared as an account of work sponsored by the United States Government. While this document is believed to contain correct information, neither the United States Government nor any agency thereof, nor The Regents of the University of California, nor any of their employees, makes any warranty, express or implied, or assumes any legal responsibility for the accuracy, completeness, or usefulness of any information, apparatus, product, or process disclosed, or represents that its use would not infringe privately owned rights. Reference herein to any specific commercial product, process, or service by its trade name, trademark, manufacturer, or otherwise, does not necessarily constitute or imply its endorsement, recommendation, or favoring by the United States Government or any agency thereof, or The Regents of the University of California. The views and opinions of authors expressed herein do not necessarily state or reflect those of the United States Government or any agency thereof or The Regents of the University of California.

\footnotetext{
*Corresponding author, email aanders@lbl.gov
} 


\title{
Measurements of the Ion Species of Cathodic Arc Plasma in an Axial Magnetic Field
}

\author{
Efim Oks ${ }^{1}$ and André Anders ${ }^{2 *}$ Fellow
}

${ }^{1}$ High Current Electronics Institute, 2/3 Academichesky Ave, Tomsk, 634055, Russia

${ }^{2}$ Lawrence Berkeley National Laboratory, 1 Cyclotron Road, Berkeley, California, 94720, USA

Abstract- Metal and gas ion species and their charge state distributions were measured for pulsed copper cathodic arcs in argon background gas in the presence of an axial magnetic field. It was found that changing the cathode position relative to anode and ion extraction system as well as increasing the gas pressure did not much affect the arc burning voltage and the related power dissipation. However, the burning voltage and power dissipation greatly increased as the magnetic field strength was increased. The fraction of metal ions and the mean ion charge state were reduced as the discharge length was increased. The observations can be explained by the combination of charge exchange collisions and electron impact ionization. They confirm that previously published data on characteristic material-dependent charge state distributions (e.g. Anders and Yushkov, J. Appl. Phys., vol. 91, pp. 4824-4832, 2002) are not universal but valid for high vacuum conditions and the specifics of the applied magnetic fields.

\footnotetext{
*Corresponding author, email aanders@lbl.gov
} 


\section{INTRODUCTION}

The term "cathodic arc" is generally used for a mode of arcs where the plasma is produced at non-stationary cathode spots [1]. A "vacuum arc" is the special case of cathodic arc when no process gas was supplied, and the latter case was extensively investigated for its relatively simplicity and ability to shed light on the cathodic processes of vacuum arcs [2-5]. When gas is added, a much greater variety of effects can happen, depending on the type of gas, gas pressure, cathode surface conditions, and the specifics of the geometry. Additionally, magnetic fields are known to play an important role because electrons are "magnetized", i.e., their trajectories are greatly influenced by the Lorentz force, and so is their ability to cause ionizing collisions [6-8].

The plasma density near the cathode spots is governed by the metal plasma produced at the spots, as opposed to the gas plasma. As gas is supplied into the discharge gap, gas plasma may become increasingly important, especially when the gas pressure exceeds about $10^{-2} \mathrm{~Pa}$. Conversely, no essential ionization of gas atoms or molecules was observed in experiment with addition of gas at low pressure [9]. With the ionization of gas it was also found that the metal plasma ions exhibited a reduced charge state as the pressure was increased. Hence, in order to obtain the highest metal charge states one must decrease the background gas pressure as much as possible [10]. There is ample and firm experimental evidence for charge-exchange as the main process responsible for reducing the metal ion mean charge state in the presence of gas neutrals [11-13].

When the cathodic arc (vacuum arc with gas feed) is combined with an external magnetic field, the ion charges states have still a tendency to be reduced by the gas, and the formation of gas ions is much promoted [11-14]. While metal ion formation occurs in the immediate vicinity of the cathode spots, gas ion generation takes place in the volume of the discharge gap [13].

In this paper, we expand on the interplay of charge exchange and volume ionization in the presence of an axial magnetic field by conducting experiments using a vacuum arc discharge ignited in an extended gap of up to $20 \mathrm{~cm}$. This work follows up on and concludes previous research done in close collaboration between the research groups in Berkeley and Tomsk (as evidenced by the collection of references given in this INTRODUCTION).

\section{EXPERIMENTAL SET-UP}

The schematic of the experimental set-up is shown in Fig. 1. It is based on the "MEVVA V” vacuum arc ion source at Lawrence Berkeley National Laboratory [15]. For the present experiments, the usual cathode unit of the ion source was replaced by a solenoid of $8 \mathrm{~cm}$ inner diameter and $20 \mathrm{~cm}$ length. A vacuum arc copper cathode (1) with the traditional high-voltage flashover trigger system was located inside the coil (6). The cathode together with the trigger ring (2) and ceramic (3) was fixed onto the end of a movable stainless steel 
rod (4). This gave us the possibility to change the location of the cathode unit within the coil from position $L=0$ to $L=20 \mathrm{~cm}$, where the zero location was chosen at the coil end facing the ion source extraction system, as indicated in Fig. 1. The inner surface of the coil body (5) and the plasma expansion housing between coil and extractions system serves as the vacuum arc anode.

Argon was selected as the working gas for its wide use in stabilizing cathodic arcs [1, 16-20]. The gas flow was provided to the coil side that is remote relative to the extraction grids, as shown in Fig. 1. The system was pumped by a cryogenic pump (nominal $1500 \mathrm{l} / \mathrm{s}$ for nitrogen) mounted to the main vacuum vessel where the time-of-flight (TOF) spectrometer was housed. The pressure in the beam analysis region was lower than in the plasma region due to differential pumping. The pressure difference between plasma and beam analysis locations was determined to be about a factor 3 since a relatively wide extraction area was used to produce a broad ion beam of merging beamlets [21-22]. The factor 3 was determined in a separate experiment (without plasma and extraction voltage) using pressure gauges on both sides of the extractor grid. The pressures mentioned in the contribution refer to the pressure in the plasma zone but are deduced from measurements downstream of the extraction system.

The traditional "Mevva V" ion source power supply and its high voltage triggering system were used to operating the cathodic arc [15]. The same arc pulse parameters were used in all experiments in terms of amplitude and duration, namely $200 \mathrm{~A}, 300 \mu \mathrm{s}$, respectively, with a pulse repetition rate of 0.3 pulses per second.

The magnetic field coil current was obtained by discharging an assembly of ten 2 $\mathrm{kV}$-capacitors in parallel with a total capacity of $250 \mu \mathrm{F}$. Triggering of the coil current was controlled by a thyristor switch. The relatively low pulse repetition rate was chosen in order to provide enough time between pulses to charge the capacitors for the magnetic coil. Measurements of the pulsed magnetic field by a small inductive pick-up probe showed that the B-field reached its maximum about $5.5 \mathrm{~ms}$ after start of the coil current. The arc triggering was therefore delayed by approximately $5.5 \mathrm{~ms}$ to utilize the maximum field. At the maximum of the coil current, the magnetic coil provided approximately $10 \mathrm{mT}$ in the center of the coil per $1 \mathrm{~A}$ of coil current.

Ions were extracted and accelerated by $40 \mathrm{kV}$ and then analyzed by a TOF spectrometer [23-24]. The current of the extracted ion beam could be measured with a Faraday cup that had magnetic self-compensation of secondary electrons. All measurements of the ion charge state distribution were made at the middle of the arc pulse, i.e., at approximately $150 \mu$ s after arc started.

\section{EXPERIMENTAL RESULTS}

The experiments showed that moving the cathode assembly within $L=0 \mathrm{~cm}$ and $20 \mathrm{~cm}$ 
did not much influence the arc burning voltage: it changed from $U_{\mathrm{b}}=26 \mathrm{~V}$ at $L=0 \mathrm{~cm}$ to $U_{\mathrm{b}}$ $=24 \mathrm{~V}$ at $L=20 \mathrm{~cm}$. The burning voltage was much more influenced by the magnetic field. Without magnetic field it was $25 \mathrm{~V}$, whereas with magnetic field it rose to $35 \mathrm{~V}$ (at $0.05 \mathrm{~T}$ ), $40 \mathrm{~V}$ (at $0.1 \mathrm{~T}$ ) and $44 \mathrm{~V}$ (at $0.2 \mathrm{~T}$ ). Moving the cathode position in the presence of the magnetic field (of $0.1 \mathrm{~T}$ ) led to greater changes of the burning voltage than in the absence of the field, namely from $28 \mathrm{~V}$ to $45 \mathrm{~V}$ for positions $0 \mathrm{~cm}$ and $20 \mathrm{~cm}$, respectively. An increase of gas pressure caused a weak reduction of the burning voltage. For example, increasing the pressure from $0.03 \mathrm{~Pa}$ to $0.1 \mathrm{~Pa}$ correlated to a decrease from $40 \mathrm{~V}$ to $36 \mathrm{~V}$.

As expected, moving the cathode far away from the extraction area had a significant influence on the extracted ion current. Moving to $L=20 \mathrm{~cm}$, the total ion beam current decreased from $90 \mathrm{~mA}$ to $16 \mathrm{~mA}$. An increase of the gas pressure caused a relatively minor decrease of the extracted ion current: the ion current fell from $44 \mathrm{~mA}$ to $25 \mathrm{~mA}$ as the argon pressure was increased from $0.03 \mathrm{~Pa}$ to $0.1 \mathrm{~Pa}$.

Figs. 2 and 3 show charge state distributions of extracted ion beams for the two extreme cases $L=0 \mathrm{~cm}$ and $L=20 \mathrm{~cm}$. One can see that the longer the path from cathode to the ion extraction zone the more gas ions appear in the ion beam. More detailed data supporting such tendency can be found in Fig. 4, which shows the composition of the extracted ion beam for different cathode positions. Another expected finding is that the decrease of the high charge state fraction of copper ions is accompanied by an increase of the fraction of lower charge states.

Feeding gas into the discharge gap and thus increasing the gas density enables the generation of more gas ions (Fig. 5).

The mean charge state of the metal ions also depends on the distance traveled from the cathode spots. The gas density is important for the reduction of the high charge state fractions of metal ions (Fig. 6): the higher the pressure the stronger the influence of the cathode position on the charge distribution of the metal ions.

We can summarize the findings as follows. Feeding gas into the discharge gap leads to two main effects. First, the presence of gas preferentially decreases the higher ion charge states and correspondingly increases the low charge states. Second, the gas enables the generation of gas ions. The appearance of gas ions is greatly determined by the presence of the magnetic field, as clearly illustrated by the experimental results of Fig. 7. Without magnetic field, only small amounts of gas ions can be found provided the cathode is positioned at a rather long distance to the extraction zone. The situation changes dramatically as soon as a rather weak magnetic field is applied.

\section{DISCUSSION}

The observations can be explained taking different types of collisions into account. In a reference frame moving with the plasma element, the density of ions of charge state $Q$ 
changes according to a balance equation of the form

$$
\begin{gathered}
\frac{\partial n_{Q}}{\partial t}=n_{e} n_{Q-1}\left\langle\sigma_{Q-1, Q}^{i o n} v_{e}\right\rangle+n_{0} n_{Q+1}\left\langle\sigma_{Q+1 \rightarrow Q}^{e x c} v_{Q+1}\right\rangle+n_{e}^{2} n_{Q+1} \alpha_{Q+1, Q} \\
-n_{e} n_{Q}\left\langle\sigma_{Q, Q+1}^{i o n} v_{e}\right\rangle-n_{0} n_{Q}\left\langle\sigma_{Q, Q-1}^{e x c} v_{Q}\right\rangle-n_{e}^{2} n_{Q} \alpha_{Q, Q-1}-\frac{n_{Q}}{\tau_{Q}}
\end{gathered}
$$

where the terms are expressed as products of the densities of particles involved and the rate coefficients of the specific type of collision. For example, in the first term $\left\langle\sigma_{Q-1, Q}^{\text {ion }} v_{e}\right\rangle$ is the rate coefficient of electron impact ionization of particles of charge state $Q-1$ leading to ions of charge state $Q$,

$$
\left\langle\sigma_{Q-1, Q}^{i o n} v_{e}\right\rangle=\int_{0}^{v_{\max }} v_{e} f\left(v_{e}\right) \sigma_{Q-1, Q}^{i o n}\left(v_{e}\right) d v_{e}
$$

in which the ionization cross section $\sigma_{Q-1, Q}^{\text {ion }}$ is folded with the velocity distribution function $f(v)$ weighted by the velocity. The positive terms of the right hand side of (1) are the processes leading to the production of particles of charge state $Q$, while the negative terms are the loss terms. The second and fifth term of (1) refer to charge exchange collisions, the third and sixth term are the three-particle recombination, the fourth term is ionization to $Q+1$, and the last terms accounts for density changes due to expansion and diffusion of particles, essentially $\nabla(D \nabla n)$ where $D$ is a diffusion coefficient.

A detailed theoretical analysis of all possible inelastic collision channels is quite involved (e.g. done for a copper hollow cathode discharge [25]) and beyond the scope of this mostly experimental contribution. However, one has to establish that the changes introduced by the gas primarily occur in the plasma zone before extraction and not in the TOF instrument in order to interpret the data in terms of plasma processes. The gas causes non-resonant charge exchange reactions of the type

$$
M^{Q}+A r \rightarrow M^{Q-1}+A r^{+} .
$$

where $M^{Q}$ stands for the $Q$-fold charged (metal) ion. Reactions of the type (3) can only occur if the energy defect is positive [12, 26], i.e. the ionization energy of the $M^{Q}$ ion is equal or greater than the ionization energy of the gas ion, here $\mathrm{Ar}^{+}$. The reaction for singly charged copper, $\mathrm{M}^{Q}=\mathrm{Cu}^{+}$, does not occur since the argon ionization energy $(15.76 \mathrm{eV})$ is greater than the first ionization energy of copper $(7.7 \mathrm{eV})$. A doubly charged copper ion however is quite effective since its ionization energy is $20.29 \mathrm{eV}$ (the excess energy of about $4.6 \mathrm{eV}$ can be found in excited states). The reduction of the higher metal ion charge states and the corresponding increase of the lower metal ion charge states is a clear "fingerprint" of 
charge exchange collisions. The reaction for triply charged copper ions $\left(M^{Q}=\mathrm{Cu}^{3+}\right)$ is more complicated since the third ionization energy is $36.84 \mathrm{eV}$, leaving an energy defect of $21.08 \mathrm{eV}$. In order for the $\mathrm{Cu}^{3+}+\mathrm{Ar} \rightarrow \mathrm{Cu}^{2+}+\mathrm{Ar}^{+}$reaction to occur, both $\mathrm{Cu}^{2+}$ and $\mathrm{Ar}^{+}$ need to be in highly excited state after the collision, or a third collision partner needs to be involved.

Most of the charge exchange processes occur in the plasma zone because (i) the gas density in the TOF instrument is lower than in the plasma zone, (ii) the cross section for charge exchange is smaller for the $80 \mathrm{keV} \mathrm{Cu}^{2+}$ ions and especially for the $120 \mathrm{keV} \mathrm{Cu}^{3+}$ ions moving through the background gas Ar than for the $\mathrm{Cu}$ ions moving with of a kinetic energy of typically 50-60 eV [7] through the Ar gas in the plasma zone [27]; (iii) the ions travel about $20 \mu$ s through the plasma zone but only about $1 \mu$ s through the TOF zone, (iv) some of the gas in the TOF path is scattered away by the beam before the measurement was done 150 $\mu$ s after beam started; and (v) the plasma zone is relative small and confined, hence gas scattering will not lead to a large reduction of gas density, quite contrary: plasma-wall interaction in the plasma zone leads to an increase of both gas and metal neutrals, and this increase is fully developed $150 \mu$ s after the plasma started [12]. Non-resonant charge exchange reactions (3) conserve energy and momentum, but the velocities of the post-collision particles are different than their pre-collision velocities, and therefore the ion that suffered a collision while traveling through the spectrometer will not arrive with those particles that traveled without collisions. The TOF peaks at the Faraday cup detector are therefore a measure for the plasma processes, although their ratio might be somewhat distorted in the spectrometer.

We further note that the presence of the magnetic field does not affect the motion of atoms, and only marginally those of ions, and hence charge exchange collisions are not sensitive to the presence of the field.

The appearance of gas ions is mainly caused by electron impact ionization. Free electrons are greatly affected by the magnetic field because their gyration radius is much smaller than the characteristic system size (here the solenoid radius). Therefore it does not surprise that the likelihood of ionization of the background gas is greatly enhanced as soon as the magnetic field is switched on. Electron impact ionization can also explain the appearance of doubly charged argon (ionization energy $40.74 \mathrm{eV}$ ) while it would take $\mathrm{Cu}^{4+}$ (ionization energy $57.38 \mathrm{eV}$ ) to produce $\mathrm{Ar}^{2+}$, yet $\mathrm{Cu}^{4+}$ has not been detected in appreciable quantities.

An increase of the path length for the metal plasma to travel through the background gas and an increase of gas density increases the likelihood of gas ionization. This tendency is much enhanced when the free electrons are magnetized. The interplay of charge exchange collisions and electron impact ionization readily explains the observed tendencies in a qualitative manner. Simulations should be done if there was a need to predict or confirm the 
results, which is beyond what we tried to demonstrate here.

\section{CONCLUSIONS}

The experimental results presented in this paper are complimentary to our previous findings that the processes of gas ionization and the reduction of metal ion mean charge states take place not at or near the cathode surface but in the space between the cathode and anode. Different collision processes with neutral atoms are responsible for this, primarily (i) charge exchange of multiply charged metal ions with gas and metal neutrals, and (ii) gas ionization by electron impact ionization facilitated by the energetic electrons of the tail of the Maxwell distribution. The presence of gas affects both types of collisions. Since electrons are readily magnetized, collision types involving free electrons are very sensitive to the absence or presence of a magnetic field.

The results are consistent with previous findings [12], namely that the charge states of metal ions are generally reduced at large distance from the spot, and thus that the metal charge states at cathode spots are higher than generally observed and reported in the literature $[2,4,7,15,28]$.

\section{ACKNOWLEDGMENTS}

We are grateful to Dr. Georgy Yushkov, HCEI Tomsk, for his suggestions and comments. We thank Joe Wallig for technical assistance. The work was supported by the IPP project LBNL-T2-0196, funded by the Department of Energy under contract AC02-05CH11231. 


\section{REFERENCES}

[1] A. Anders, Cathodic Arcs: From Fractal Spots to Energetic Condensation. New York: Springer, 2008.

[2] V. M. Lunev, V. G. Padalka, and V. M. Khoroshikh, "Plasma properties of a metal vacuum arc. II," Sov. Phys. Tech. Phys., vol. 22, pp. 858-861, 1977.

[3] H. C. Miller, "Measurements on particle fluxes from dc vacuum arcs subjetced to artificial current zeros," J. Appl. Phys., vol. 43, pp. 2175-2181, 1972.

[4] J. Kutzner and H. C. Miller, "Integrated ion flux emitted from the cathode spot region of a diffuse vacuum arc," J. Phys. D: Appl. Phys., vol. 25, pp. 686-693, 1992.

[5] R. L. Boxman, D. M. Sanders, and P. J. Martin, Eds., Handbook of Vacuum Arc Science and Technology. Park Ridge, N.J.: Noyes Publications, 1995.

[6] E. M. Oks, A. Anders, I. G. Brown, M. R. Dickinson, and R. A. MacGill, "Ion charge state distributions in high current vacuum arc plasma in a magnetic field," IEEE Trans. Plasma Sci., vol. 24, pp. 1174-1183, 1996.

[7] A. Anders and G. Y. Yushkov, "Ion flux from vacuum arc cathode spots in the absence and presence of magnetic fields," J. Appl. Phys., vol. 91, pp. 4824-4832, 2002.

[8] M. Galonska, R. Hollinger, and P. Spadtke, "Charge sensitive evaluated ion and electron energy distributions of a vacuum arc plasma," Rev. Sci. Instrum., vol. 75, pp. 1592-1594, 2004.

[9] P. Spädtke, H. Emig, B. H. Wolf, and E. Oks, "Influence of gas added to the MEVVA discharge on the extracted ion beam," Rev. Sci. Instrum., vol. 65, pp. 3113-3118, 1994.

[10] G. Y. Yushkov and A. Anders, "Physical limits for high ion charge states in pulsed discharges in vacuum," J. Appl. Phys., vol. 105, pp. 043303-5, 2009.

[11] A. Anders and G. Y. Yushkov, "Puzzling differences in bismuth and lead plasmas: evidence for the significant role of neutrals in cathodic vacuum arcs," Appl. Phys. Lett., vol. 91, p. 091502, 2007.

[12] A. Anders, E. M. Oks, and G. Y. Yushkov, "Production of neutrals and their effects on the ion charge states in cathodic vacuum arc plasmas," J. Appl. Phys., vol. 102, p. 043303, 2007.

[13] E. Oks, G. Y. Yushkov, and A. Anders, "Temporal development of ion beam mean charge state in pulsed vacuum arc ion sources," Rev. Sci. Instrum., vol. 79, p. 02B301, 2008.

[14] B. H. Wolf, H. Emig, D. M. Rück, P. Spädtke, and E. Oks, "A Mevva ion source for simultaneous implantation of gas and metal ions," Nucl. Instrum. Meth. Phys. Res. B, vol. 106, pp. 417-428, 1995.

[15] I. G. Brown, "Vacuum arc ion sources," Rev. Sci. Instrum., vol. 65, pp. 3061-3081, 1994. 
[16] C. Hwang, E. Row, N. Shi, and E. Sun, "Method for depositing a thin film adhesion layer," US Patent 25045468, 2005.

[17] M. Kühn, R. Pintaske, and F. Richter, "Optical emission spectroscopy in cathodic arc deposition," IEEE Trans. Plasma Sci., vol. 25, pp. 694-699, 1997.

[18] F. Richter, G. Krannich, J. Hahn, R. Pintaske, M. Friedrich, S. Schmidbauer, and D. R. T. Zahn, "Utilization of cathodic arc evaporation for the deposition of boron nitride thin films," Surf. Coat. Technol., vol. 90, pp. 178-183, 1997.

[19] J. Vetter, W. Burgmer, and A. J. Perry, "Arc-enhanced glow discharge in vacuum arc machines," Surf. Coat. Technol., vol. 59, pp. 152-155, 1993.

[20] Y. J. Zhang, P. X. Yan, Z. G. Wu, W. W. Zhang, G. A. Zhang, W. M. Liu, and Q. J. Xue, "Effects of substrate bias and argon flux on the structure of titanium nitride films deposited by filtered cathodic arc plasma," physica status solidi (a), vol. 202, pp. 95-101, 2005.

[21] I. G. Brown, Ed., The Physics and Technology of Ion Sources. Weinheim: Wiley-VCH, 2004.

[22] A. T. Forrester, Large Ion Beams. New York: Wiley, 1988.

[23] I. G. Brown, J. E. Galvin, R. A. MacGill, and R. T. Wright, "Improved time-of-flight charge state diagnostic," Rev. Sci. Instrum, vol. 58, pp. 1589-1592, 1987.

[24] V. I. Gushenets, A. G. Nikolaev, E. M. Oks, L. G. Vintizenko, G. Y. Yushkov, A. Oztarhan, and I. G. Brown, "Simple and inexpensive time-of-flight charge-to-mass analyzer for ion beam source characterization," Rev. Sci. Instrum., vol. 77, p. 063301, 2006.

[25] A. Bogaerts and R. Gijbels, "Hybrid modeling network for a helium--argon--copper hollow cathode discharge used for laser applications," J. Appl. Phys., vol. 92, pp. 6408-6422, 2002.

[26] B. H. Bransden and M. R. C. McDowell, Charge Exchange and the Theory of Ion-Atom Collisions. Oxford, UK: Clarendon Press, 1992.

[27] T. P. Grozdanov and R. K. Janev, "Charge-exchange collisions of multiply charged ions with atoms," Phys. Rev. A, vol. 17, pp. 880-888, 1978.

[28] A. A. Plyutto, V. N. Ryzhkov, and A. T. Kapin, "High speed plasma streams in vacuum arcs," Sov. Phys. JETP, vol. 20, pp. 328-337, 1965. 


\section{Figure Captions}

Fig.1. Experimental set-up based on the "Mevva V" ion source with modified cathode unit; the numbered labels have the following meaning: 1: copper cathode, 2: triggering ring, 3: ceramic flashover ring, 4: movable stainless steel rod, 5: anode, 6: magnetic field coil, 7: emission (plasma) grid, 8: high voltage insulator: 9: ion extraction grids.

Fig.2. Ion beam mass/charge spectra for cathode position $0 \mathrm{~cm}, B=0.05 \mathrm{~T}$, argon $p=0.04$ Pa.

Fig.3. Ion beam mass/charge spectra for cathode position $20 \mathrm{~cm}, B=0.2 \mathrm{~T}$, argon $p=0.04$ Pa.

Fig.4. Composition of the extracted ion beam at different cathode positions. Arc current 200 A, argon, $p=0.04 \mathrm{~Pa}, B=0.2 \mathrm{~T}$.

Fig.5. Total fraction of metal (copper) ions in extracted beam at different cathode positions. Arc current $200 \mathrm{~A}, B=0.2 \mathrm{~T}$.

Fig. 6. Metal (copper) ion mean charge state in extracted beam at different cathode position. Arc current $200 \mathrm{~A}, B=0.2 \mathrm{~T}$.

Fig. 7. Total fraction of gas ions (Ar $p=0.04 \mathrm{~Pa}$ ) in the extracted beam for different cathode positions. Arc current $200 \mathrm{~A}$. 


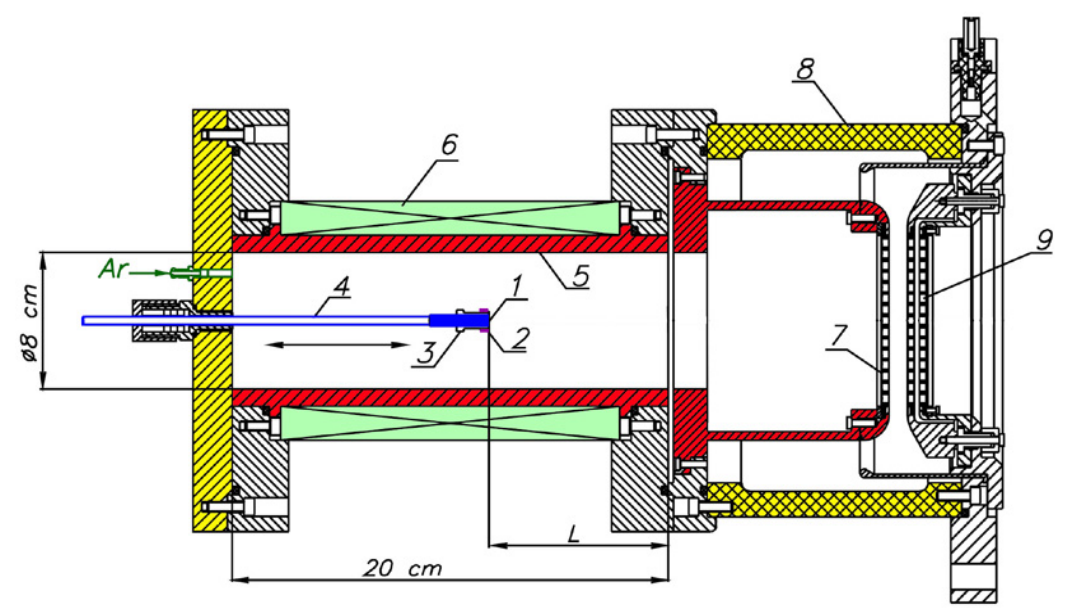

Fig. 1 


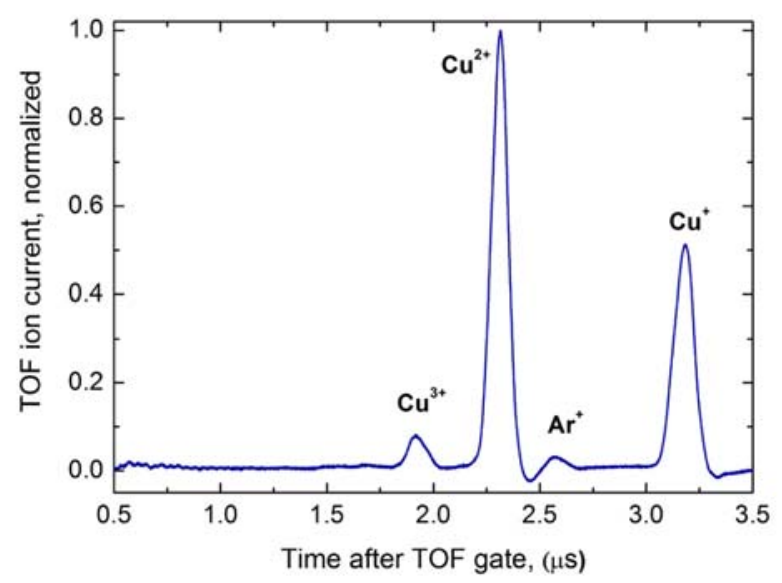

Fig. 2 


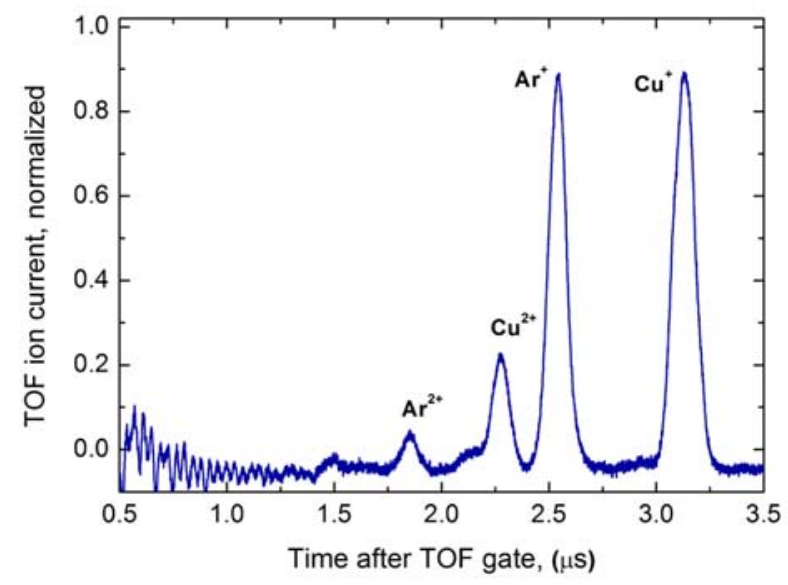

Fig. 3 


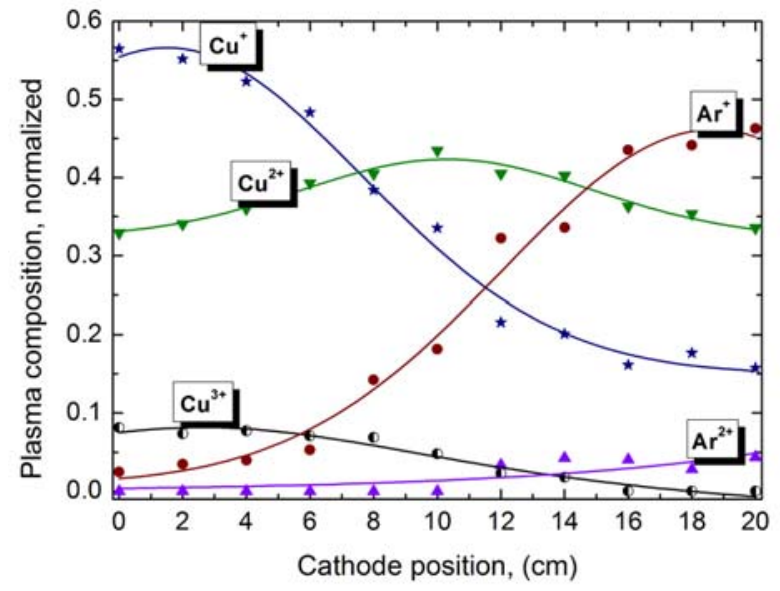

Fig. 4 


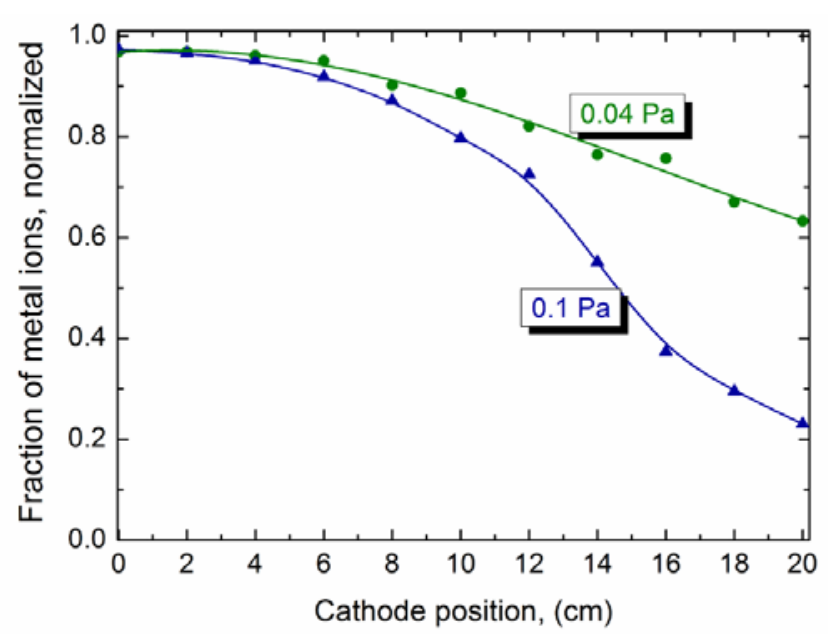

Fig. 5 


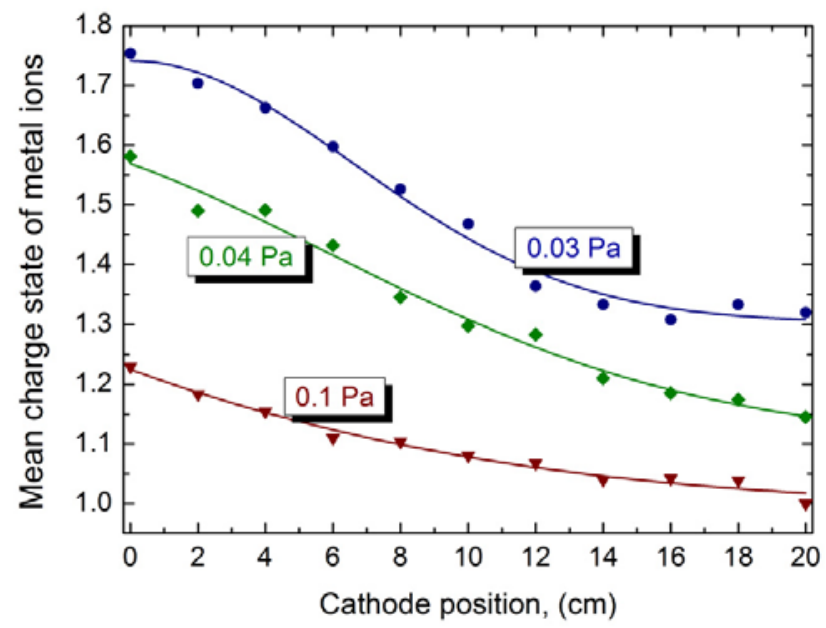

Fig. 6 


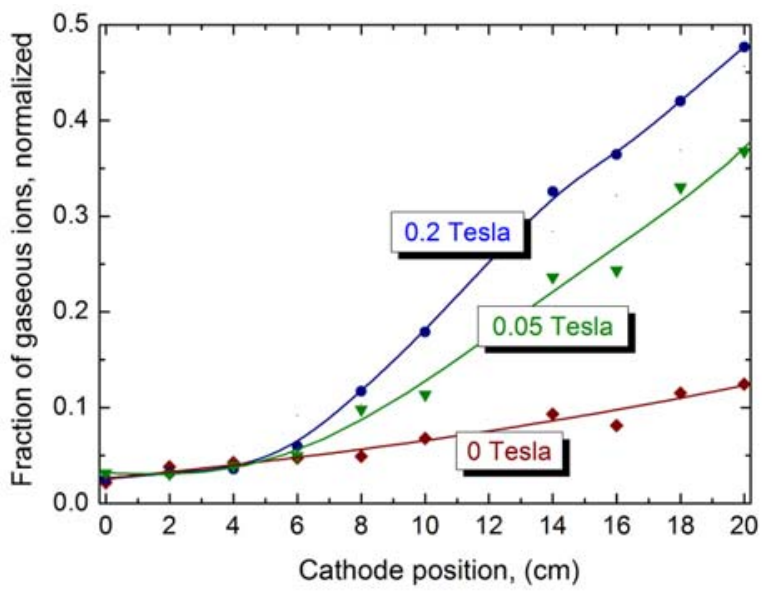

Fig. 7 